\title{
Association of Sleep Quality with Socio- Demographic Characteristics in Elderly Referred to Health Centers in Qazvin, Iran
}

\author{
Leila Dehghankar ${ }^{1}$, Azam Ghorbani ${ }^{\star}{ }^{\star}$, Leili Yekefallah ${ }^{3}$, Marziyeh Hajkarimbaba ${ }^{4}$, Akram Rostampour ${ }^{5}$ \\ ${ }^{1}$ Instructor of Nursing, Department of Community Health Nursing, School of Nursing \& Midwifery, Qazvin University of Medical Sciences, Qazvin, Iran \\ ${ }^{2}$ Instructor of Nursing ,Metabolic Diseases Research Center and School of Nursing \& Midwifery ,Qazvin University of Medical Sciences, Qazvin, Iran \\ ${ }^{3}$ Assistant Professor, PhD of nursing, Faculty of Nursing, School of Nursing \& Midwifery, Qazvin University of Medical Sciences, Qazvin, Iran \\ ${ }^{4}$ Nursing, Qazvin University of Medical Sciences, Qazvin, Iran \\ ${ }^{5}$ Student of Nursing, Student Research Committee, Qazvin University of Medical Sciences, Qazvin, Iran
}

\begin{abstract}
Background and aim: Sleep disorders are not innately harmful to quality of life, they can induce or complicate physical and mental sicknesses and increase the risk of mortality. The aim of this study was to determine the association of sleep quality components with socio-demographic characteristics in elderly people in Qazvin, Iran.

Materials and Methods: This descriptive cross-sectional study was performed on 400 elderly patients referred to health centers in Qazvin. Data were collected by using a demographic questionnaire and the Pittsburgh sleep quality index (PSQI). Data were analyzed by SPSS 19 software, descriptive and inferential statistics (Mann-Whitney, Chi-square, logistic regression). Results: The prevalence of poor sleep quality was $80 \%$. Statistically significant difference were observed between total global PSQI score and economic condition $(p<0.03)$ and had chronic physical disease $(P<0.001)$. Highest component scores was sleep latency $(1.55 \pm 1.06)$ and lowest was habitual sleep efficiency $(0.16 \pm 0.57)$. Older women had worse sleep quality compared with older men $(P<0.05)$. Multivariate logistic regression model indicated that female sex is associated with greater tendency towards poor sleep quality $(\mathrm{OR}=1.78,95 \% \mathrm{Cl}=1.08-2.94, \mathrm{P}=0.022)$.

Conclusion: This study provides evidences that poor sleep quality was high among the elderly of Qazvin. It seems sleep quality assessment of older adults is necessary to identify the factors affecting sleep quality and to adopt appropriate measures and strategies to eliminate or reduce the effect of these factors.

Keywords: elderly, sleep quality, community health centers
\end{abstract}

\section{INTRODUCTION}

Sleep is a basic need of human being because it assists the body to relax and repair, maintain appropriate circadian rhythm and preserve energy for daily living (Azri et al., 2016). Insufficient sleep can increase fatigue and

\footnotetext{
*Correspondence: ghorbani_az@yahoo.com

Azam Ghorbani, Metabolic Diseases Research Center, Velayat Hospital, 22 Bahman BLvd, Minoodar, Qazvin, Iran. Phone: +98 2833790620 Fax: +9828 333790611

Received: 10 October 2017 Accepted: 31 October 2017
}

Sleep and Hypnosis

Journal homepage:

www.sleepandhypnosis.org

ISSN:1302-1192 (Print) 2458-9101 (Online) unrestrained daytime sleepiness. Sleep complications have an adverse effect on mental and physical health, impair the quality of life and increase the healthcare expenses. Quality of sleep will finally influence energy, emotional balance and health. Therefore, poor sleep quality and sleep disorders such as insomnia can undermine the quality of life (Azri et al., 2016). As a result of aging, changes such as decreased efficiency and total sleep time and increased superficial sleep take place, thereby influencing the quantity and quality of sleep (Mendoza-Meléndeza et al., 2016).

Sleep disorders in old age are prevalent and have been known (Wu, Tung-Ping Su, Chin-Lung Fang, \& Mei Yeh Chang, 2012). Approximately $50 \%$ of the adults aged 
$>65$ are sleep disorders and have been reported at least one chronic sleep complaint, the most common being inability to stay asleep at night (Chein \& Chen, 2015, Landry, Best, \& Ambrose, 2015, Smyth, 2008). So that, Park et al. reported $60 \%$ of the elderly had poor sleep quality (Park, Yoo, \& Bae, 2013). Sleep disorders in the elderly can be caused by various reason, such as medical disorders, atypical sleep-wake patterns, reduced amount of sleep or poor sleep quality. it should be noted that so Sleep disorders have resulted in the poor quality of life , including induced or complicate physical and mental sicknesses and increase the risk of mortality (Smagula, Stone, Fabio, \& Cauley, 2016);besides Poor sleep quality in old age is caused by environment, chronic disease, pain and sleep disturbance and too contributes cardiac illness, depression, falls and accidents. Recent findings show sleep quality plays a key role in maintaining cognitive function in elderly (Landry, Best, \& Ambrose, 2015). Therefore, it is highly important to consider sleep quality and to identify sleep disorders among the elderly.

Knowledge the quality of sleep in the elderly and identification of the factors affecting it will be helpful to adopt appropriate strategies to wipe out or decrease the impact of these factors. However, there is very little, inadequate and indecisive data regarding sleep quality and sleep quality predictors among older people with different levels of cognitive functioning in an institution in Iran. Hence, this study was conducted to determine the association of sleep quality components and sociodemographic characteristics in elderly people in Qazvin, Iran.

\section{METHOD}

This descriptive survey was performed on a representative sample of community-based individuals aged 60 years and older in Qazvin city, Iran. For calculating sample size were applied the following formula:

$n=\frac{\left(Z_{1-\alpha} / 2\right)^{2} * P(1-P)}{d^{2}}=\frac{4 * 0.5(1-0.5)}{(0.05)^{2}}=\frac{1}{0.0025}=400$

In this formula Considering $\mathrm{p}$ as prevalence of sleep problems (49.4\%)(10) (Nazifi et al., 2014); Test confidence and test power are considered respectively; $95 \%$ and 0.05 , the least number of the samples was estimated 400 were selected by multistage cluster random sampling method based on the number of elderly population in an urban health center. Participants aged 60 years or over (Noroozian, 2012), ability for verbal communication to response the questions and without a history of cognitive and mental diseases and disability were included to the study; so written informed consent was gained from all participants. For data gathering all participants were interviewed face to face by trained two nurses at health center. Those who older participants were not available for interview at their home for another appointment. Because elderly people have at least one chronic disease such as arthritis, high blood pressure, Diabetes, cardiovascular diseases, Musculoskeletal disorders, Neurological diseases, sensory disorders and ... (Barry, 2000), (Pishkar Mofrad, Jahantigh, Arbabisarjou, 2015) Chronic disease of the participants were recorded using medical disease list. The scale applied to measure sleep quality was the Pittsburgh sleep quality index (PSQI) (Smyth, 2008; Nazifi et al., 2014). The PSQl is a validated tool that contains 19 questions on multiple dimensions that assess sleep quality and problems in the past month. This questionnaire consist of seven components score on a 0-3 scale: subjective sleep quality, sleep latency, sleep duration, habitual sleep efficiency, sleep disturbances, use of sleep medication and day time dysfunction. The sum of scores of the above seven component scores presents a global PSQ score with a scope of 0-21 points. A lower global PSQI score showed good sleep quality and a global score PSQI score higher than 5 was defined as poor sleep quality (Buysse et al., 1991).

Data were recorded as mean \pm standard deviation (SD) or number (percent). The PSQI factors were compared between male and females using Mann Whitney $U$ test. Chi-square test was used for analysis of categorical variables. A logistic regression analysis was used to examine the association of sleep status and demographic variables. P-values less than 0.05 were considered as statistically significant. 


\section{RESULTS}

Of 400 older participants, 194 (48.5\%) were males and 206 (51.5\%) were females. Mean age was $67.48 \pm$ 7.09 years, The majority of the subjects were in the 60-65 age groups, , married (71.5\%); Among the participant, $51.8 \%$ were living with wife, and. Only $18 \%$ had received a high school education or above. Most (84.5\%) of the subjects had a chronic physical disease. With regard to economic condition, $87.8 \%$ were Independent.

The total global PSQI score was $7.11 \pm 3.04$. The prevalence of poor sleep was $80 \%$. Mean of sleep duration was $5.81 \pm 1.61$ hours in males and $5.79 \pm 1.76$ in females. Comparison of the sleep qualities of the older subjects and their socio-demographic characteristic are presented in table 1 . Statistically significant difference were observed between total global PSQ score and economic condition $(p<0.03)$ and had chronic physical disease $(P<0.001)$. There was no statistically significant difference between the total global PSQI score and age, education, marital status living status and duration of menopauses.

Total PSQI scores and its seven components of the study subjects by sex are shown in table 2; as shown in the table highest component scores was sleep latency $(1.55 \pm 1.06)$ and lowest was Habitual sleep efficiency $(0.16 \pm 0.57)$. Women older had worse sleep quality compared with older men $(P<0.05)$. No significant differences were found between males and females in sleep duration, habitual sleep efficiency, sleep disturbances and use of sleep medication. Female older had longer sleep latency, and more day time dysfunction. Multivariate logistic regression model indicated that female sex is associated with greater tendency towards poor sleep quality $(\mathrm{OR}=1.78,95 \% \mathrm{Cl}=1.08-2.94$, $\mathrm{P}=0.022$ ), no association was found between age, education, disease or living condition and poor sleep quality (table 1) (table 2).

Table 1: Mean Scores of PSQI according to demographic variables of the elderly subjects

\begin{tabular}{|c|c|c|c|}
\hline & \multicolumn{2}{|c|}{ Total PSQI Score } & \multirow[b]{2}{*}{ P-Value } \\
\hline & n (\%) & Mean \pm SD & \\
\hline \multicolumn{4}{|l|}{ Marital status * } \\
\hline Single & $114(28.5)$ & $7.33 \pm 3.67$ & \multirow[b]{2}{*}{0.10} \\
\hline Married & $286(71.5)$ & $6.51 \pm 3.18$ & \\
\hline Age * & & $7.01 \pm 3$ & \\
\hline $60-65$ & $206(51.5)$ & & \\
\hline $66-70$ & $94(23.5)$ & $6.87 \pm 3.01$ & \\
\hline $71-75$ & 47 (11.8) & $7.82 \pm 3.21$ & \\
\hline $76-80$ & $27(6.8)$ & $7.66 \pm 2.98$ & 0.45 \\
\hline$>80$ & $26(6.5)$ & $6.88 \pm 3.12$ & \\
\hline \multicolumn{4}{|l|}{ Education * } \\
\hline Illiterate & $121(30.2)$ & $7.28 \pm 2.97$ & \multirow{4}{*}{1.67} \\
\hline Primary & $154(38.6)$ & $7.47 \pm 2.94$ & \\
\hline High school & $53(13.2)$ & $7.04 \pm 3.81$ & \\
\hline Academic & $72(18)$ & $6.45 \pm 3.03$ & \\
\hline \multicolumn{4}{|l|}{ Living condition * } \\
\hline Alone & $57(14.2)$ & $7.80 \pm 3.59$ & \multirow{4}{*}{0.44} \\
\hline With wife & $207(51.8)$ & $6.92 \pm 2.83$ & \\
\hline With children & $133(33.2)$ & $7.13 \pm 3.09$ & \\
\hline With relatives & $3(0.8)$ & $6 \pm 0.0$ & \\
\hline \multicolumn{4}{|l|}{ Disease ** } \\
\hline Yes & $338(84.5)$ & $7.35 \pm 3.04$ & \multirow{2}{*}{$<0.001$} \\
\hline NO & $62(15.5)$ & $5.77 \pm 2.63$ & \\
\hline \multicolumn{4}{|l|}{ Economic situation** } \\
\hline Independent & $351(87.8)$ & $7 \pm 3.04$ & \multirow{2}{*}{0.03} \\
\hline Dependent upon others & 49 (12.2) & $7.91 \pm 2.92$ & \\
\hline
\end{tabular}

*Kruskal Wallis Test, **Mann Whitney 
Dehghankar / Sleep and Hypnosis 2018

Table 2. Total PSQI scores and seven component scores of the elderly subjects by sex

\begin{tabular}{lccccc}
\hline Component & Total & Men & Women & Z value & P-value \\
\hline Subjective sleep quality & $1.10 \pm 0.69$ & $1.05 \pm 0.72$ & $1.15 \pm 0.65$ & -1.579 & 0.114 \\
Sleep latency & $1.55 \pm 1.06$ & $1.45 \pm 1.04$ & $1.64 \pm 1.06$ & -1.753 & 0.080 \\
Sleep duration & $1.74 \pm 0.97$ & $1.71 \pm 0.98$ & $1.77 \pm 0.97$ & -.666 & 0.505 \\
Habitual sleep efficiency & $0.16 \pm 0.57$ & $0.15 \pm 0.56$ & $0.16 \pm 0.58$ & -.322 & 0.747 \\
Sleep disturbances & $1.43 \pm 0.57$ & $1.40 \pm 0.57$ & $1.45 \pm 0.58$ & -.745 & 0.457 \\
Use of sleep medication & $0.51 \pm 1.06$ & $0.50 \pm 1.05$ & $0.53 \pm 1.07$ & -.395 & 0.693 \\
Daytime dysfunction & $0.59 \pm 0.81$ & $0.52 \pm 0.77$ & $0.67 \pm 0.85$ & -1.867 & 0.062 \\
Total PSQI & $7.11 \pm 3.04$ & $6.80 \pm 2.98$ & $7.39 \pm 3.07$ & -1.903 & 0.057 \\
\hline
\end{tabular}

\section{DISCUSSION}

Sleep complications have an adverse effect on mental and physical health, impair the quality of life and increase the healthcare expenses (Azri et al., 2016). This study evaluated the sleep quality of the elderly of Qazvin, Iran.

The findings showed $80 \%$ of the elderly had poor sleep quality. These results were in line with the findings of Park et al. (Park, Yoo, \& Bae, 2013), Oliviera et al (Oliviera Araújo \& Ceolim, 2010) and Azri et al (Azri et al., 2016), indicating $64.3 \%$ of the Korean elderly, $63.1 \%$ of the Brazilian elderly and the Indonesian elderly had poor sleep quality, respectively (Azri et al., 2016). However, Niu et al reported that $33.7 \%$ of the Chinese elderly had poor sleep quality (Niu et al., 2016). King Lee et al. (Lee et al., 2015) and Wu et al. (Wu, Tung-Ping Su, Chin-Lung Fang, \& Mei Yeh Chang, 2012). Reported $37.6 \%$ and $49 \%$ of the elderly had poor sleep quality, respectively, which was much lower than the results of the present study in terms of the incidence of poor sleep quality. It seems poor sleep quality is quite prevalent in the elderly of Qazvin, Iran. It should be noted that physiologic changes due to aging affect the sleep quality of the elderly negatively. Yet, the contradictory results of studies can be associated with the self-report data collection method and uncontrollable psychological conditions of the elderly while completing the questionnaires.

The results of the current study showed that the elderly in the subgroup "Sleep latency" had maximum level of mean sleep quality and those in the subgroup "Habitual sleep efficiency" had minimum level of mean sleep quality. In their study, Park et al reported maximum level of mean sleep quality for the component scores "sleep latency" and "sleep duration" and minimum level of mean sleep quality for the component scores "use of sleep medication"; Further, the obtained results indicated that the sleep quality of the elderly was reduced with aging, but no statistically significant difference was observed, confirming the results of Niu et al (Niu et al., 2016), Kiejna et al (Kiejna, Rymaszewska, Wojtyniak, \& Stokwiszewski, 2004) and Wu et al (Wu, Tung-Ping Su, Chin-Lung Fang, \& Mei Yeh Chang, 2012). The findings of Park et al. for the Korean elderly (Park, Yoo, \& Bae, 2013) and those of Jeong Park (Jeong Park, 2017) was conflict. The findings also demonstrated a significant difference between men and women in total global PSOI score, sleep quality being poorer in women than in men. This was in agreement with the results of Smagula et al (9) (Smagula, Stone, Fabio, \& Cauley, 2016), Wu et al (Wu, Tung-Ping Su, Chin-Lung Fang, \& Mei Yeh Chang, 2012) and Niu et al (Niu et al., 2016). However, the study of Park et al. on the Korean elderly population showed no significant relationship between gender and sleep quality (Park, Yoo, \& Bae, 2013).

Moreover, the results indicated a significant difference between sleep quality and chronic disease status of the elderly, so that with an increase in chronic diseases in the elderly, their sleep quality was worsened, which was in line with the findings of Hsiu (Hsiu \& Lin, 2005), Niu et al (Niu et al., 2016) and Wu et al (Wu, Tung-Ping Su, ChinLung Fang, \& Mei Yeh Chang, 2012). Further, Park et al stated that chronic disease in the elderly was a predictor of their sleep quality (Park, Yoo, \& Bae, 2013). Furthermore, the findings of the present study showed a significant association between sleep quality and economic status of the elderly; people with higher income had a better sleep quality. It is quite evident that 
higher income is a factor that reduces the mental and psychological concerns of the elderly. Adams (Adams, 2006), Friedman (Friedman et al., 2007) and Smagula (Smagula, Stone, Fabio, \& Cauley, 2016) emphasized the positive effect of income on solving the sleep disorders.

Our results indicated that the elderly with lower education had poorer sleep quality, but there was no significant difference between education status of the elderly and their sleep quality. Yet, the cross-sectional study of Niu et al on 1010 Chinese elderly showed a significant relationship between education level and sleep quality al (Niu et al., 2016). It should be noted that the positive effect of education on sleep quality has been formerly reported by various researchers. (Jeong Park, 2017, Wu, Tung-Ping Su, Chin-Lung Fang, \& Mei Yeh Chang, 2012; Kiejna, Rymaszewska, Wojtyniak, \& Stokwiszewski, 2004; Moore, Adler, Williams, \&Jackson; 2002).

The current results also showed no significant difference between marital status of the elderly and their sleep quality, confirming the findings of Wu et al (Wu, Tung-Ping Su, Chin-Lung Fang, \& Mei Yeh Chang, 2012), Park et al (Park, Yoo, \& Bae, 2013) and Smagula (Smagula, Stone, Fabio, \& Cauley, 2016). It is noteworthy that married people are supported by their families, but the single ones are deprived of this kind of support, which consequently causes stress and sleep problems. Meanwhile, divorce is one of the risk factors involved in sleep disorders (Lee et al., 2015). Additionally, the findings revealed no significant difference between sleep quality and duration of menopause among the female elderly. However, Hsiu et al (Hsiu \& Lin, 2005) reported menopause played a key role in structural changes of elderly women's sleep, and they suffered sleep disorder after menopause.

The self-report of the questionnaire regarding the elderly's sleep quality was one of the limitations of this study, as a result of which the psychological and uncontrollable factors could affect their responses. Small sample size was another limitation, which could undermine the generalizability of the findings. Further research with larger sample size in all healthcare centers of Qazvin province can help to generalize the obtained results.

\section{CONCLUSION}

The results were indicative of poor sleep quality among the elderly of Qazvin province, Iran. Since sleep is one of the basic needs of human being, any disorder in sleep causes mental problems and loss of personal abilities (Smagula, Stone, Fabio, \& Cauley, 2016), it seems necessary to identify the factors affecting sleep quality among the elderly and to adopt appropriate measures and strategies to eliminate or reduce the effect of these factors. It should be noted that planning to face the challenges of poor sleep quality in the elderly is a vital step to be taken, and lack of planning and policymaking in this regard will result in serious social, economic and health problems for the society in the near future. Since promotion of sleep hygiene in the elderly is potentially important, special programs are recommended to be developed to enhance the social participation, improve medical and health services and present counselling services to the elderly population.

\section{References}

Adams, J. (2006). Socioeconomic position and sleep quantity in UK adults. Journal of Epidemiology and Community Health; 60(3): 267-9. doi: 10.1136/jech.2005.039552.

Araújo, C. L. D. O., \& Ceolim, M. F. (2010). Sleep quality of elders living in long-term care institutions. Revista da Escola de Enfermagem da USP, 44(3), 619-626.

Azri, M.A., Dahlan, A., Ghazali, Masuri, M., Anuar, Md, Isa KH(2016). Sleep Quality among Older Persons in Institutions. Procedia Social and Behavioral Sciences, 234:74-82. https://doi. org/10.1016/j.sbspro.2016.10.221.

Barry P. P (2000). An overview of special considerations in the evaluation and management of the geriatric patient. The American Journal of Gastroenterology; 95:8-10. http://dx.doi. org/10.1111/j.1572-0241.2000.01697.x;)

Buysse, D.J., Reynolds, C.F. 3rd, Monk, T.H., Hoch, C.C., Yeager, A.L., Kupfer, D.J. (1991).Quantification of subjective sleep quality in healthy elderly men and women using the Pittsburgh Sleep Quality Index (PSQI). Sleep. 14(4):331-8.

Chein, M.Y., Chen, H.C (2015). Poor Sleep Quality is Independently Associated with Physical Disability in Older Adults. Journal of Clinical Sleep Medicine; 11(3):225-232. doi: 10.5664/jcsm.4532 
Friedman, E.M., Love, G.D., Rosenkranz, M.A., Urry, H.L., Davidson, R.J., Singer, B.H. Ryff C.D. (2007). Socioeconomic status predicts objective and subjective sleep quality in aging women. Psychosomatic Medicine, 69(7):682-91. DOI:10.1097/ PSY.0b013e31814ceada

Hsiu, H.C., Lin, M.H. (2005). Exploring quality of sleep and its related factors among menopausal women. Journal of Nursing Research, 13(2):153-164. doi: 10.1097/01. JNR.0000387536.60760.

Park, M. J., \& Kim, K. H. (2017). What affects the subjective sleep quality of hospitalized elderly patients?. Geriatrics \& Gerontology International, 17(3), 471-479. doi: 10.1111/ ggi.12743.

Kiejna, A., Rymaszewska, J., Wojtyniak, B., Stokwiszewski, J. (2004). Characteristics of sleep disturbances in Poland - results of the National Health Interview Survey. Acta Neuropsychiatrica, 16(3):124-9. doi: 10.1111/j.0924-2708.2004.00063.x.

Landry, G.J., Best, J.R., Ambrose, T.L. (2015). Measuring sleep quality in older adults: a comparison using subjective and objective methods. Frontiers in Aging Neuroscience 7, 1-10. doi:10.3389/fnagi.2015.00166.

Lee, L.Y., Tam, K.W., Lee, M.L., Lau, N.Y., Lau, J.C., Lam, Y.M., Lam, C.H., Kwan, W.K., Chan, D., Chan, B., Chan, P.Z. (2015). Sleep quality of middle-aged Tai Chi practitioners. Japan Journal of Nursing Science, 12(1):27-34. doi: 10.1111/jjns.12045.

Mendoza-Meléndez, M. Á., Jimenez-Correa, U., Gallegos-Cari, A., Ayala-Guerrero, F., \& Jiménez-Anguiano, A. (2016). Prevalence of sleep disorders, daytime sleepiness and clinical symptomatology in older adults. Revista Médica del Hospital General de México, 79(3), 136-143. Doi: 10.1016/j. hgmx.2016.05.021

Moore, P.J., Adler, N.E., Williams, D.R., Jackson, J.S. (2002). Socioeconomic status and health: the role of sleep. Psychosomatic Medicine, 64(2):337-44.
Nazifi, M., Mokarami, H., Akbaritabar, A., Kalte, A.O., Rahi, A. (2014). Psychometric Properties of the Persian Translation of Pittsburgh Sleep Quality. Health Scope, 3(2): 1-4.

Niu, J., Han, H., Wang, Y., Wang, L., Gao, X., Liao, S. (2016). Sleep quality and cognitive decline in a community of older adults in Daqing City, China. Sleep Medicine 17, 69-74. https://doi. org/10.1016/j.sleep.2015.07.033.

Noroozian M (2012). The Elderly population in Iran: An ever growing concern in the health system. Iranian Journal of Psychiatry and Behavioral Sciences, 6(2): 1-6. PMCID: PMC3940007

Park, J.H., Yoo, M.S., Bae, S.H. (2013). Prevalence and predictors of poor sleep quality in Korean older adults. International Journal of Nursing Practice, 19, 116-23. doi: 10.1111/ijn.12047.

Mofrad, Z. P., Jahantigh, M., \& Arbabisarjou, A. (2016). Health Promotion Behaviors and Chronic Diseases of Aging in the Elderly People of Iranshahr*-IR Iran. Global journal of health science, 8(3), 139-45. doi: 10.5539/gjhs.v8n3p139

Smagula, S.F., Stone, K.L., Fabio, A., Cauley, J.A. (2016). Smagula Risk factors for sleep disturbances in older adults: Evidence from prospective studies. Sleep Medicine Reviews, 25, 21-30. doi: 10.1016/j.smrv.2015.01.003.

Smyth, C.A. (2008). Evaluating sleep quality in older adults: the Pittsburgh Sleep Quality Index can be used to detect sleep disturbances or deficits. American Journal of Nursing, 108(5):42-50? doi: 10.1097/01.NAJ.0000317300.33599.63.

Wu, C.Y., Tung-Ping, Su. B., Chin-Lung, Fang, C, Mei, Yeh, Chang, A. (2012). Sleep quality among community-dwelling elderly people and its demographic, mental, and physical correlates. Journal of the Chinese Medical Association, 75, 75-80. https:// doi.org/10.1016/j.jcma.2011.12.011 